\title{
IKAT: A TOOL FOR INCREMENTAL DEVELOPMENT OF ONTOLOGIES THROUGH VERSIONS
}

\author{
Mikel Larrañaga, Jon A. Elorriaga \\ University of the Basque Country
}

\begin{abstract}
In this paper an ontology-based knowledge acquisition tool is presented. This work is focused on the incremental development of ontologies and aims to help with the creation and maintenance of knowledge-based systems. The tool uses the version concept to implement the evolution of the ontologies. IKAT facilitates the development of ontologies through versions propagating automatically changes to the knowledge bases built based on them. An analysis of the possible operations upon ontologies has been carried out, and as a result a set of plausible operations has been chosen and their treatment implemented.
\end{abstract}

Key words: Knowledge acquisition, ontologies, knowledge engineering, reusability.

\section{INTRODUCTION}

Ontologies, which had been used in other areas such as philosophy, were adopted to represent the knowledge in order to minimize the communication difficulties in KBS development teams. In knowledge engineering area, ontologies are defined as " an explicit specification of a conceptualization" [3]. They provide a formalism for the representation of a concrete domain that can be understood without any ambiguity either by different people or by a computer. Obviously, a broadly accepted representation of the domain is hardly reached immediately, so the process of building ontology must be gradual and requires several refinements.

Many ontology-based tools appeared with the aim of relieving the mentioned bottleneck by minimizing the knowledge engineers role as they allow domain experts to transfer their knowledge to a computer system even though they are not familiar with computers. Some of these tools are Protégé- 
2000 [1], Ontolingua [2] and Kads22 [5]. However, most of these tools lack of facilities to incrementally enhance ontologies. SHOE language [4] allows versioning of ontologies but it does not automate the updating of $\mathrm{KBs}$ built based on them. The work here presented focuses on the incremental development of ontologies and presents IKAT. The aim of this tool is to allow the refinement and modification of ontologies and to automatically propagate changes to concrete $\mathrm{KBs}$.

The paper starts presenting IKAT design issues. Next the architecture of IKAT is detailed and the last section focus on how IKAT helps with the incremental ontology development by using versions. Finally we present some conclusions and future research lines.

\section{DESIGN AND IMPLEMENTATION}

IKAT is a general propose knowledge acquisition tool that aids to constructing knowledge-based systems; in order to allow the gradual ontology definition in an intuitive way, IKAT uses the version concept. A version of an ontology includes the ontology itself and the KBs built based on it. New versions of an ontology can be created by experts on the following circumstances:

- While the ontology is being developed: An ontology will go through several phases until a consensus is reached. Each of these phases can be a version.

- As an improvement of the original ontology: When the domain changes, the ontology that describes it also must change, e.g., a production system describing ontology must update itself if a new product or a production way appears.

- As an adaptation of the ontology to a particular application: An ontology that describes completely a domain may have too much information for a simple application, e.g., a car domain describing ontology can include even the car production process, but there are a lot of entities and relations that an expert diagnose tool of the electrical system does not need.

- As an enhancement of existing KBSs: User can extend the ontology a KBS is based on in order to exchange information with others KBS.

IKAT distinguishes two different phases in the ontology building process in the way Protégé does: the definition of the abstract objects of the domain (classes) and the definition of the concrete objects (instances). IKAT allows building several knowledge bases of an ontology for particular applications. 


\section{ARCHITECTURE OF IKAT}

Figure 1 shows the architecture of IKAT. The kernel of IKAT is composed by two functional modules (Ontology Acquisition Module and Knowledge-Base Acquisition Module), which exchange information with the Knowledge-Base Library information repository. Each of the components of the IKAT architecture will be described below. The architecture of IKAT has been designed in three layers in order to enhance its changeability. The Ontology Acquisition Module, the Knowledge Acquisition Module and the KB Library are part of the kernel layer. Communication Interface is on the middle layer and it communicates the top layer (graphic interface) with the kernel layer. Communication Interface allows IKAT to be used in the two different modes: as an autonomous tool through the graphic interface or used by another application through the communication interface.

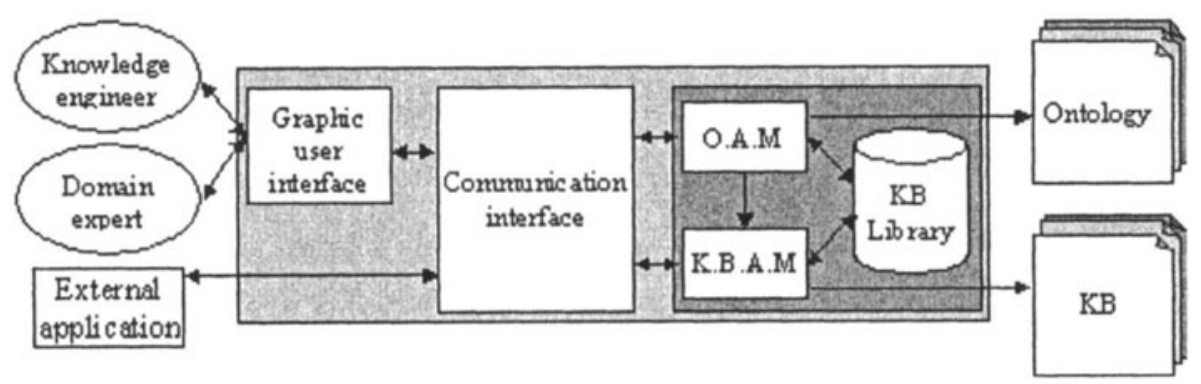

Figure 1. Architecture of IKAT

\subsection{Ontology Acquisition Module (OAM)}

On this module, the user creates the classes, attributes and relations among classes that describe the domain are created. This module is the responsible of building and managing the ontologies. Once the ontology has been completely defined, it generates the executable code that will be used in knowledge based system, and stores the internal representation so that it can be used to create new version or even to update the current ontology.

\subsection{Knowledge Base Acquisition Module (KBAM)}

On this module, the user defines the instances of each of the KBs based on the ontology that has been built on the OAM. This module is the responsible of building the KBs based on the ontology. As Figure 1 illustrates, this module communicates with the Ontology Acquisition module. 
This message interchange is because some of the operations applied to the ontology imply some updates in its knowledge bases.

\subsection{Communication Interface}

It contains the complete set of high-level functions defined for IKAT. These functions can be used either by the graphic interface or by any external application.

\subsection{Graphic Interface}

IKAT defines three working levels:

- General management level: IKAT has a set of windows that allows the user to create, refine and delete ontologies and knowledge bases as well as to create new versions. IKAT represents by a tree structure the relations among the ontologies and their versions and another one to organize each ontology's knowledge bases. When a version is open or created control is passed to the next level.

- Ontology management level: At this level, IKAT helps the user to construct and modify ontologies. Every time, the state of the ontology is represented by a graph in two modes, a simple one that just shows the class hierarchy, and a richer one which shows the classes with their attributes. Once the ontology is defined, the user can go through to the next level and define the knowledge bases. Figure 2 shows the interface for this level.

- Knowledge base management level: Another tree structure is used in this level to show the defined instances and classes.

\subsection{Knowledge-Base Library}

This is the responsible of storing and retrieving the created ontologies and knowledge bases together with their versions for future use. As it is shown on Figure 1, both functional modules (OAM and KBAM) generate an output file. OAM generates a file containing the code implementing the abstract objects of the domain; KBAM produces a file with the defined instances of each KB. Joining these files, we get the skeleton of the desired knowledge based system. IKAT uses an internal object-oriented representation language for the ontologies and KBs. 


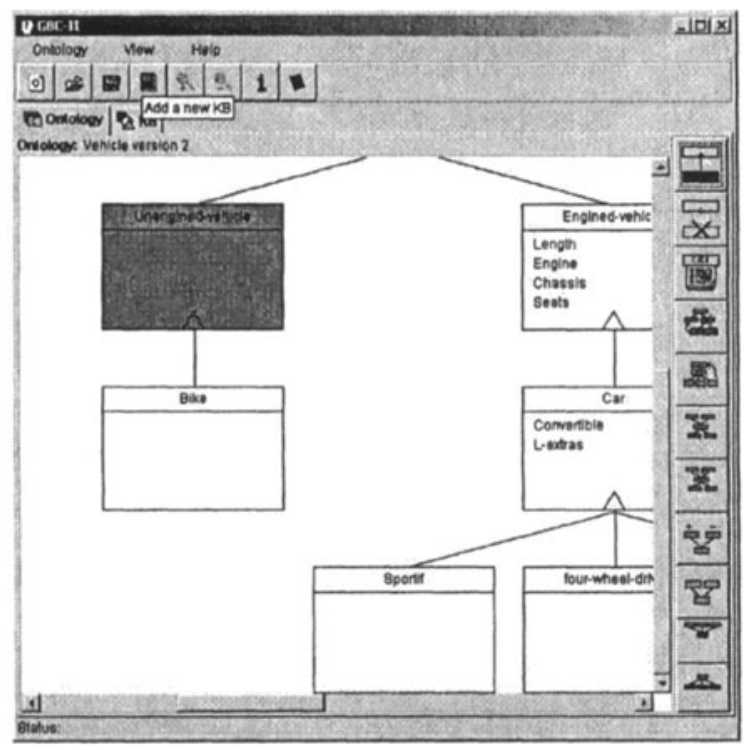

Figure 2. Ontology Management Level

\section{INCREMENTAL DESIGN IN IKAT}

In order to promote the incremental design of ontologies and KBs, IKAT provides versioning facilities and automatic propagation of changes in complex operations on ontologies.

\subsection{Ontology versioning}

As it has been mentioned above, building an ontology is not a simple process, but it requires successive improvements. This ontology refining is facilitated in IKAT by generating successive versions of it, and thus, reusing the represented knowledge. A version is generated by making a copy of the whole ontology and all the KBs based on it. Besides, the reference to the original copy to the original ontology is stored. Therefore, IKAT stores all the versions of the ontology in a structured way. As it will be mentioned in next section, any operation is propagated to KBs by IKAT adapting them to the changes that happen on the ontology. Therefore, IKAT quickens the process of building knowledge based systems by facilitating the updating of the ontologies they use to represent the domain. Propagations of operations through versions could be performed that way. 


\subsection{Automatic propagation of operations to KBs}

In order to enhance IKAT with operation that will facilitate ontology development, an analysis of the operations that can be applied to ontologies has been carried out. All this operations can be performed on ontologies, so the changes they provoke must be propagated to the KBs built based on them. Therefore, complex operations that hardly could be performed on KBs without rebuilding them have been included in this analysis. Once most important operations have been identified, they have been analysed obtaining the effects they entail on the ontology and its KBs In addition, several treatments have been proposed and among them the appropriate treatment has been chosen taking into account the following criteria: (1) automatic propagations must be promoted in order to lighten the work, (2) constructive operations are directly performed, and (3) the destructive operations will demand user confirmation. In some cases and in order to maximize the automatic propagation of the changes, IKAT proposes a different way to make the operation.

Besides the basic operations such as creating a new class or instance, IKAT provides the following complex operations with the aim of facilitating the incremental development of ontologies/KBs by automating the propagation of changes. These propagations can happen in two different levels: (1) on the ontology due to the inheritance, such as propagating an attribute or removing from descendants an inherited attribute, or (2) on KBs so as to keep the consistency among the ontology and the KBs built based on it.

Table 1 and Table 2 summarize the most relevant operations that have been analysed. These tables present the operations and their effects on the ontology and the proposed treatments. In addition, the effects on KBs are detailed taking into account each treatment. The selected treatment of the operation is shown shadowed. Reasons for choosing a particular treatment are also specified in these tables by its criteria number.

\subsubsection{Operations on Ontologies}

- Class Specialization: Specialization can be defined as the creation of a set of disjoint subclasses according to one or several attributes. The individual objects of the specialized class will be relocated into the subclasses according to the values of the attributes that compose the disjunction criteria. To perform this operation, a leaf class, i.e., a class without any subclasses, must be selected.

- Generalization of a set of classes that have at least a common ancestor: This operation needs that the classes share at least a common 
ancestor. IKAT allows two generalization modes:

- A type generalization: In this type of generalization, a new class will replace the set of classes. The new class contains only the common attributes of all the classes that are included in the operation and will be subclass of all their common ancestors. Their subclasses will descend from the new class. Due to this operation, the descendants of the selected classes will not inherit non-common attributes anymore. Therefore, those attributes will be also removed from instances of the selected classes and their descendants.

- B type Generalization: IKAT generates a new ancestor for the set of selected classes that includes their common attributes. These attributes will be inherited from the selected classes, so it is not necessary to represent them in the subclasses. In this case, instances are not really affected because there are neither new attributes nor redefinitions

- Modifying the defined class-superclass relations: IKAT allows creating new class-superclass relations (New class superclass relation), removing defined relations (Remove class superclass relation) and even replacing any superclass (Change superclass) by another one. Precedence order, i.e. the priority among classes, that determines from which superclass an attribute is inherited if it is defined in more than one, can also be modified (Change Precedence order). Adding a new class-superclass relation may cause some attributes to appear in the class and its subclasses and also another ones to be redefined. Changing class-superclass relation may also cause some attributes to be modified, new attributes to appear and other ones to be deleted. Removing a class-superclass relation may cause some attributes redefinitions and some others to be removed. When an attribute is removed from a class it is also removed from any of its instances or any descendant that inherited it. When an attribute is redefined, the value it has on instances is replaced by its new default value. When a new attribute is propagated it is initialized on instances with its default value.

- Class removing operations: IKAT provides three different operations for removing classes.

- A type Class Removal: The subclasses of the removed class will be related directly to the ancestors of the removed class. Instances of the descendants of the removed class will not inherit its attributes anymore. Besides, as classes have changed their ancestors, some of the attributes may be redefined and, thus, they will change their value. IKAT will look for the new value/s on the class that propagates the attribute.

- B type Class Removal: The subclasses of the removed class will be from now on descendants of the brother class (i.e., a class with a common ancestor) the user has chosen as new parent. Instances will suffer several changes on their attributes, i.e., some attributes will be 
removed, new ones propagated and some others will change their value. IKAT will use the default values that are defined on the new parent to initialize the new attributes and to modify the ones that will be redefined.

Table 1. Operations and their effects on the ontology

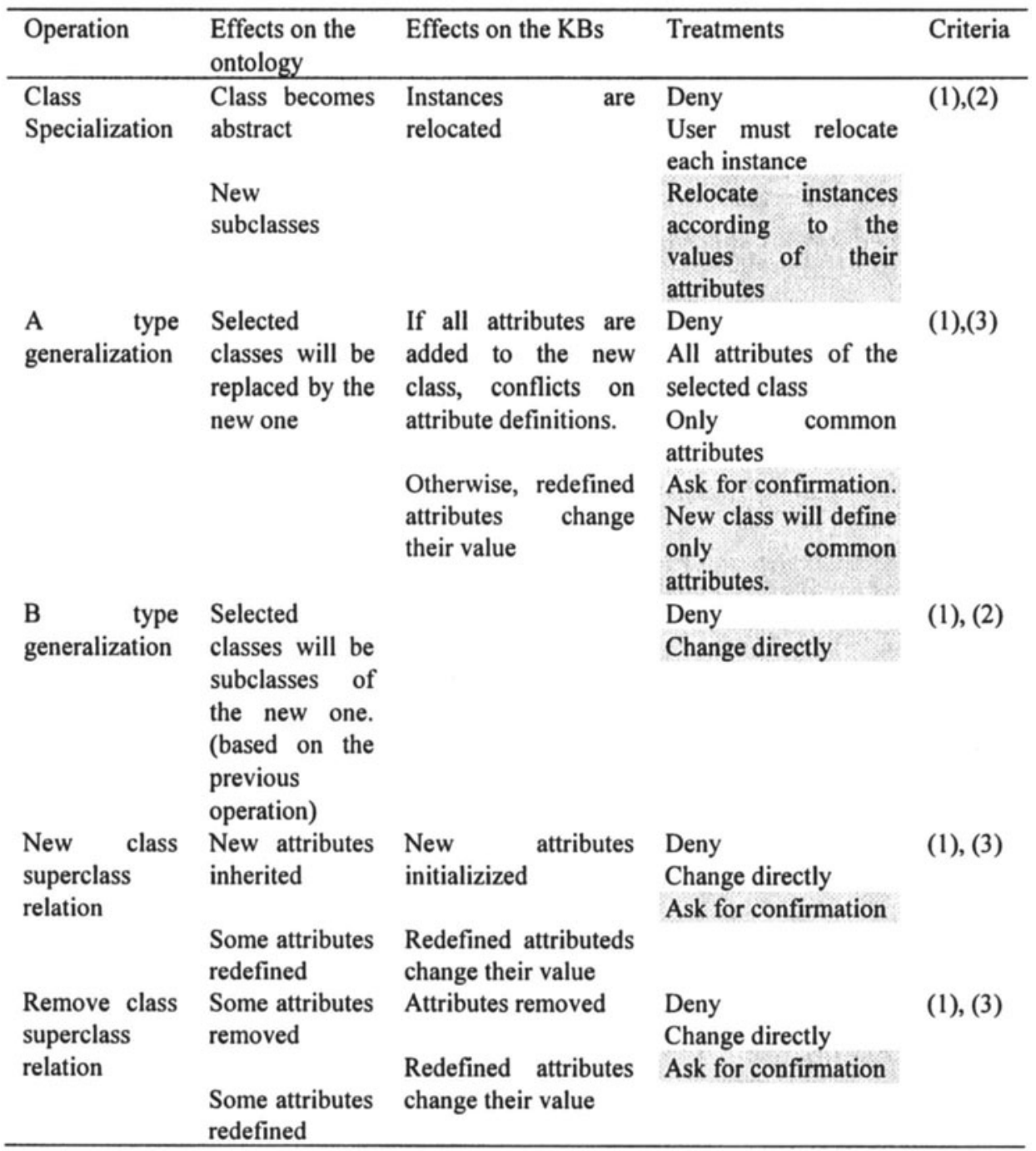

- C type Class Removal: IKAT allows removing classes in a recursive way. This operation is propagated through the hierarchy by deleting the descendants of the removed class if it is their only ancestor or deleting the class-superclass relation in other case. As a result of this operation, instances of removed classes will be deleted. Instances of the modified 
descendant classes will suffer attribute removals and modifications; thus, the modified attributes will take their value/s from the next priority parent.

Table 2. Operations on ontologies and their effects

\begin{tabular}{|c|c|c|c|c|}
\hline Operation & $\begin{array}{l}\text { Effects on the } \\
\text { ontology }\end{array}$ & Effects on the KBs & Treatments & Criteria \\
\hline $\begin{array}{l}\text { Class } \\
\text { Specialization }\end{array}$ & $\begin{array}{l}\text { Class becomes } \\
\text { abstract } \\
\text { New } \\
\text { subclasses }\end{array}$ & $\begin{array}{l}\text { Instances } \\
\text { relocated }\end{array}$ & $\begin{array}{l}\text { Deny } \\
\text { User must relocate } \\
\text { each instance } \\
\text { Relocate instances } \\
\text { according to the } \\
\text { values of their } \\
\text { attributes }\end{array}$ & $(1),(2)$ \\
\hline $\begin{array}{l}\text { A type } \\
\text { generalization }\end{array}$ & $\begin{array}{l}\text { Selected } \\
\text { classes will be } \\
\text { replaced by the } \\
\text { new one }\end{array}$ & $\begin{array}{l}\text { If all attributes are } \\
\text { added to the new } \\
\text { class, conflicts on } \\
\text { attribute definitions. } \\
\text { Otherwise, redefined } \\
\text { attributes change } \\
\text { their value }\end{array}$ & $\begin{array}{l}\text { Deny } \\
\text { All attributes of the } \\
\text { selected class } \\
\text { Only common } \\
\text { attributes } \\
\text { Ask for confirmation. } \\
\text { New class will define } \\
\text { only common } \\
\text { attributes. }\end{array}$ & $(1),(3)$ \\
\hline $\begin{array}{l}\text { B type } \\
\text { generalization }\end{array}$ & $\begin{array}{l}\text { Selected } \\
\text { classes will be } \\
\text { subclasses of } \\
\text { the new one. } \\
\text { (based on the } \\
\text { previous } \\
\text { operation) }\end{array}$ & & $\begin{array}{l}\text { Deny } \\
\text { Change directly }\end{array}$ & $(1),(2)$ \\
\hline $\begin{array}{l}\text { New class } \\
\text { superclass } \\
\text { relation }\end{array}$ & $\begin{array}{l}\text { New attributes } \\
\text { inherited } \\
\text { Some attributes } \\
\text { redefined }\end{array}$ & $\begin{array}{l}\text { New attributes } \\
\text { initializized } \\
\text { Redefined attributeds } \\
\text { change their value }\end{array}$ & $\begin{array}{l}\text { Deny } \\
\text { Change directly } \\
\text { Ask for confirmation }\end{array}$ & (1), (3) \\
\hline $\begin{array}{l}\text { Remove class } \\
\text { superclass } \\
\text { relation }\end{array}$ & 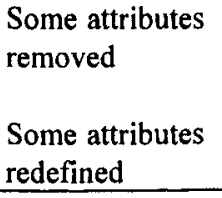 & $\begin{array}{l}\text { Attributes removed } \\
\text { Redefined attributes } \\
\text { change their value }\end{array}$ & $\begin{array}{l}\text { Deny } \\
\text { Change directly } \\
\text { Ask for confirmation }\end{array}$ & (1), (3) \\
\hline
\end{tabular}

- Modifying class properties: IKAT permits modification of the properties of the class. For example, the user can determine if instances of the class can be defined or not. If the user establishes that a class can not have any instance, IKAT will delete all its instances 
- Modifying attribute properties: As well as class properties can be modified, IKAT allows redefining the properties of attributes that were specified while creating it. For example, the type of value and the cardinality of the attribute can be modified. If the type of value is changed, the value of the instances will be replaced by its new default value. If cardinality of the attributes is reduced to a single value, the instances will only keep the first value they had on the attribute.

- Removing attributes: Also attributes can be removed. As a result of this operation, the attribute will be removed from every instance that mentions any of the affected classes.

All the operations that have been described in this section involve the instances that are defined on each of the KB built based on the modified ontology. IKAT takes into account the modifications the ontology has suffered and automatically updates its knowledge bases.

\subsubsection{Operations on KBs}

As classic operations on KBs, such as defining new instances, do not entail any problem, they will not be mentioned in this section:

- Relocating instances in other classes: IKAT allows relocating instances in other classes and it automatically removes all the no more needed slots, adds the new ones and updates the ones that are redefined giving them default values.

- Removing instances: IKAT allows the removal of instances from the knowledge bases too. IKAT maintains the relations between instances, which is updated as an instance is removed.

\section{BENEFITS OF USING IKAT}

In order to show the advantages IKAT provides to the user, the amount of operation it saves to the user will be quantified. The major benefits of using IKAT are obtained with complex operations on ontologies where slots removals and redefinitions are propagated through the ontology and the KBs. Constructive ontology operations and knowledge base operations are already supported by other similar. systems so they are not taken into account. However, in those systems, side effects like slot removals and redefinitions due to complex destructive operations on ontologies are not automatically performed. This can cause problems when trying to load a knowledge base after modifying the ontology it is based on, so the user should perform this changes on instances manually before using the KB. As it has been explained above, IKAT's complex operations are refined into a sequence of basic 
operations such as slot redefinitions and slot removals. Therefore, the number of updates the user should perform manually in other systems can be calculated with the following formula:

Updates $=$ AffInst $* B O$

Where Updates represents the number of modifications the user should perform, AffInst is the number of affected instances and $B O$ is the number of basic operations that must be performed. $B O$ is quite easy to obtain (see Table 1 and Table 2), but in order to get the number of modified instances the ontology should be carefully analysed because slot redefinitions can be found in any descendant class. Therefore, the formula here presented calculates the number of instance updates in the worst case:

$$
\begin{aligned}
& \text { AffInst }=\operatorname{Inst}(c)+\operatorname{InstDesc}(c) \\
& \operatorname{InstDesc}(C)=\sum_{i=1}^{S C N(C)}\left(\operatorname{Inst}\left(\operatorname{sc}_{i}(\mathrm{C})\right)+\operatorname{InstDesc}\left(s C_{i}(C)\right)\right) \\
& \operatorname{Inst}(c)=\sum_{j=1}^{K B N} I\left(K B_{j}, C\right)
\end{aligned}
$$

Inst(C): Number of instances of $C$ class

InstDesc (C): Number of instances of the descendants of $C$ class

$\operatorname{SCN}(C)$ : Number of subclasses of $C$ class

SCi(C): i-th Subclass of C class

KBN: Number of knowledge bases based on the ontology

It is impossible to specify with a general formula the number of instance updates that can happen on an ontology because there is no way to describe on it when a class redefines a slot. Therefore, this recursive formula will be illustrated with a sample ontology so as to get an idea of how much work IKAT could save to the user. The class hierarchy of this ontology has a binary tree structure in order to ease the calculation of the number of affected classes and instances. Let's suppose that $X \mathrm{KBs}$ have been defined based on this sample ontology and $K$ instances have been defined for each class on each knowledge base; if there are $P$ levels from the modified class to the bottom of the hierarchy then the number of instances can be represented by the following formula:

$$
\text { Inst }=\left(1+\sum_{i=1}^{p} 2^{i}\right) * K^{*} X
$$




\section{CONCLUSIONS}

In this paper, IKAT, a tool that supports the full life cycle of an ontology, has been described. IKAT graphic interface allows the knowledge engineer or domain expert both to define an ontology by specifying its abstract objects, and to construct the Knowledge Base (KB) creating and relating instances. The interface releases the user from knowing the language implementation details.

Several tools for editing ontologies have been developed. IKAT contributes in this area providing a means for incremental ontology development through versions. An ontology is constructed in a gradual process involving a team of experts. However, during this process KBs can be developed based on the ontology or a fragment of it. Therefore, a knowledge acquisition tool should support the management of different versions of an ontology. Also, in order to lighten the workload of updating the ontologies and the Knowledge Bases (KBs) based on them, such a tool should maximize the automatic propagations of the changes in the ontologies and knowledge bases. IKAT was designed with these aims.

\section{ACKNOWLEDGMENTS}

The works presented in this paper are supported by the University of the Basque Country (UPV/EHU) (1/UPV 00141.226-T-13946/2001), the Economy Department of the Gipuzkoa Council (IRIS-D(II); RF 949/2001), the Basque Government (UE1999/36) and the CICYT (TIC99-0252).

\section{REFERENCES}

[1] Eriksson, H., Fergerson, R. W., Shahar, Y. and Musen, M. A. Automatic Generation of Ontology Editors. Twelfth Banff Knowledge Acquisition for Knowledge-based systems Workshop, Banff, Alberta, Canada. 1999.

[2] Farquhar, A., Fikes, R. and Rice, J. The ontolingua server: Tool for collaborative ontology construction. IJHCS, 46(6): 707--728. 1997.

[3] Gruber, T. R. A translation approach to portable ontology specifications. Knowledge Acquisition 5, 199--220. 1993.

[4] Heflin, J. and Hendler, J. Dynamic ontologies on the Web. Proceedings of the Seventh National Conference on Artificial Intelligence (AAAI-2000). AAAI/MIT Press, Menlo Park, CA, pp. 443-449. 2000.

[5] Kabel, S. and Anjewierden, A. An introduction to KADS22 functionality. http://www.swi.psy.uva.nl/projects/kads22/help/intro/intro.htm. 1999 